\title{
Microscopic study of vorticities in relativistic chiral fermions
}

\author{
Kiyoumars A. Sohrabi \\ Albert Einstein Center for Fundamental Physics, University of Bern, \\ Sidlerstrasse 5, 3012 Bern, Switzerland \\ E-mail: sohrabi@itp.unibe.ch
}

ABSTRACT: It is usually believed that unlike the external magnetic field which one can set directly, vorticity is a property of the flow of particles, which is indirectly controlled by external fields and initial conditions. Using the curved-space technics it is shown that the influence of the vorticity on the relativistic chiral fermions can indeed be controlled directly.

KEYworDS: Effective field theories, Chiral Lagrangians

ArXiv EPRINT: 1409.4528 


\section{Contents}

1 Introduction 1

2 Review of the tools $\quad 4$

2.1 Local Lorentz frames 4

2.2 The equations of motion 5

3 Applications $\quad 6$

$\begin{array}{ll}3.1 & \text { Vortical dipole moment }\end{array}$

$\begin{array}{ll}3.2 \text { Dirac equation } & 8\end{array}$

$\begin{array}{lll}3.3 & \text { Modified Lorentz generators } & 10\end{array}$

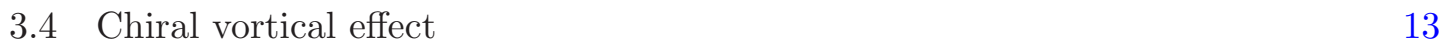

4 Summary 14

\section{Introduction}

Field theories under the influence of external sources have been studied since a long time ago. One important application of the quantization of these theories goes back to Schwinger in 1951 [1]. He considered the effect of background electromagnetic fields on $\gamma$-decay of neutral mesons. His technics were developed further and got various applications in relativistic hadron-hadron collisions [2], QCD phenomenology using chiral effective Lagrangians [3] and gravitational anomalies [4]. As such is chiral magnetic effect (CME), introduced by Kharzeev et al. [5-9]. This effect occurs when a nonzero current is produced along the magnetic field in a chiral medium. Separate computations to approximate its size has been done [10] and comparison with observables such as charge-dependent correlations [11, 12] provided a good foundation for further analysis.

A closely related exciting problem to the CME is chiral vortical effect (CVE) where a current appears along the axis of vorticity. Historically, it was first realized by Vilenkin in $1980[13,14]$, while complete understandings of the hydrodynamical current and its connection to the triangle anomaly was given by Son and Surowka [15]. It has been studied in the context of gauge/gravity duality [16, 17], non-zero chemical potential [18], group theory [19], kinetic theory [20, 21] and topological invariants in momentum space [22]. Its applications are numerous; in principle all phenomena that include magnetic fields, influence of vorticities can also be implemented into the medium. Future application of the CVE will shed light on 3D graphene and Weyl semimetals [23-25]. Further relevant topics in this context are superfluids and Fermi liquids [26-31].

Although a lot has been done in the literature to understand CME in the recent years, but the same treatment is lacking in the context of CVE. For a few computations 
for instance refer to [32-36]. In fact, there are many contradictions about these anomalyinduced effects and their physical picture [37]. For a review on related issues refer to [38, 39]. We show using standard methods that CVE is comprehensible in the context of quantum field theory and deducible from first principles self-consistently.

First, let's mention an issue with causality; the common definition of the vorticity from classical mechanics is $\boldsymbol{\omega}=\frac{1}{2} \nabla \times \boldsymbol{u}$. Consider fluid's motion confined to a plane in two dimensions. Then $\omega_{z}=\frac{1}{r} \frac{\partial}{\partial r}\left(r u_{\phi}\right)$, where $(r, \phi)$ are $2 \mathrm{D}$ cylindrical coordinates. We are looking for a setup that the vorticity is zero at the origin at some initial time thus we choose $u_{\phi}=a r^{n}$. Then $\omega_{z}=a(n+1) r^{n-1}$ for $n>1$. That is, the vorticity gets larger as we go further away from the origin and eventually fluid's speed becomes larger than the speed of light.

One way to overcome this problem is to promote the Minkowski's flat spacetime to a curved spacetime. To see this, note that the Poincaré group leaves the Minkowski metric in flat space invariant. Infinitesimal transformation of this group has the form, $y^{\prime a}=y^{a}+\xi^{a} \epsilon$. Invariance of the Minkowski metric then means that $\partial_{c} y^{\prime a} \partial_{d} y^{\prime} b \eta^{c d}=\eta^{a b}$. The key point is that an arbitrary boost of the form $x^{\mu}=x^{\mu}\left(y^{\prime}\right)$ satisfies $\xi^{\mu}(x)=\partial_{a^{\prime}} x^{\mu} \xi^{\prime}\left(y^{\prime}\right)$. Under this transformation the Minkowski metric is no longer invariant $g^{\mu \nu}=\partial_{a^{\prime}} x^{\mu} \partial_{b^{\prime}} x^{\nu} \eta^{a b}$.

In recent years, applications of curved spacetime backgrounds as a probe for symmetries of the underlying flat-spactime field theories have become popular. The basic idea is very simple, intuitively whenever we want to study the effect of electromagnetism, we add gauge potentials to the Lagrangians of the field theories. Similarly, the study of a system under relativistic rotations or vorticities is possible by a specific choice of a background metric. In what follows, we consider perturbative background perturbations of the type $g_{\mu \nu}(x)=\eta_{\mu \nu}+h_{\mu \nu}(x)$. Generally, we can classify these attempts in two groups of macroscopic and microscopic physics.

The macroscopic physics. Perturbative technics with the background metric have already been used for deriving Kubo formulas in the relativistic hydrodynamics [40]. As an example, it has been shown that in a conformal parity-even theory, the hydrodynamic transport coefficient that couples vorticities in the stress tensor is given by

$$
T^{\mu \nu}=T_{\text {ideal }}^{\mu \nu}-\lambda_{3} \Omega_{\lambda}\left\langle\mu \Omega^{\nu\rangle \lambda},\right.
$$

with $\Omega^{\mu \nu} \equiv \frac{1}{2} \Delta^{\mu \alpha} \Delta^{\nu \beta}\left(\nabla_{\alpha} u_{\beta}-\nabla_{\beta} u_{\alpha}\right)$, if for simplicity we assume the shear tensor, $\sigma^{\alpha \beta} \equiv$ $2 \nabla^{\langle\alpha} u^{\beta\rangle}=0$. Here we are using the convenient notation, $A^{\langle\mu \nu\rangle} \equiv \frac{1}{2} \Delta^{\mu \alpha} \Delta^{\nu \beta}\left(A_{\alpha \beta}+A_{\beta \alpha}\right)-$ $\frac{1}{3} \Delta^{\mu \nu} \Delta^{\alpha \beta} A_{\alpha \beta}$, that subtracts the trace of the tensor if we choose $\Delta^{\mu \nu}=g^{\mu \nu}+u^{\mu} u^{\nu}$. The above information is sufficient to determine the corresponding Kubo formula [41, 42],

$$
\lambda_{3}=-4 \lim _{p^{z}, q^{z} \rightarrow 0} \partial_{p^{z}} \partial_{q^{z}} G_{E}^{x y, x t, y t}(p, q)
$$

where here $G_{E}^{x y, x t, y t}$ is defined in [43] as

$$
G_{\mathrm{E}}^{x y, x t, y t}(p, q) \equiv \int d^{4} x d^{4} y e^{-i(p \cdot x+q \cdot y)} \times\left.\frac{2^{3} \partial^{3} \ln \mathrm{Z}}{\partial g_{y t}(x) \partial g_{x t}(y) \partial g_{x y}(0)}\right|_{g_{\mu \nu}=\delta_{\mu \nu}},
$$


with

$$
Z\left[g_{\mu \nu}\right]=\int \mathcal{D} \phi \exp \left\{-S_{\mathrm{E}}\left[\phi, g_{\mu \nu}\right]\right\}
$$

which basically what it's saying is that, $\lambda_{3}$ is given by the second derivatives of the threepoint correlation function of the stress tensors plus some contact terms. For a derivation of this transport coefficient directly from partition function refer to [44, 45] or [46]. The lesson to take home from the above example is that specific choice of the metric components, in this case $h_{0 x}(z)$ and $h_{0 y}(z)$, gives nonzero values for $\Omega_{x z}=-\frac{1}{2} \partial_{z} h_{o x}$ and $\Omega_{y z}=-\frac{1}{2} \partial_{z} h_{o y}$.

In parity-odd theories, on the other hand, vorticity as an axial vector appears at first order in the derivative expansion in the current $j^{\mu}[15]$,

$$
j^{\mu}=n u^{\mu}-\sigma T\left(g^{\mu \nu}+u^{\mu} u^{\nu}\right) \partial_{\nu}\left(\frac{\mu}{T}\right)+\xi \omega^{\mu}, \quad \omega^{\mu}=\epsilon^{\mu \nu \lambda \rho} u_{\nu} \Omega_{\lambda \rho},
$$

and the Kubo formula for the anomalous coefficient $\xi$, reads as,

$$
\xi=-i \lim _{p^{y} \rightarrow 0} \partial_{p^{y}} G_{E}^{t y, z}(p),
$$

where the above Euclidean Green's function is expressed in terms of the second derivatives of the partition function. The first derivative is with respect to $A^{z}$, the $z$ component of the electromagnetic four-potential, then the second derivative with respect to $g^{t y}$. This works since as one can check, the lower-indexed rest frame will be $u_{\mu}=\left(-1, h_{o x}, 0,0\right)$. Plugging this value into the definition of the vorticity, we obtain $\omega^{z}=-\frac{1}{2} \partial_{y} h_{0 x}$.

The microscopic physics. With a self-consistent approach to study the relativistic vorticity, we will be capable of uncovering new properties of field theories in various mediums. The most significant examples are fermionic systems in which calculations are naturally more involved. For simplicity, we concentrate on massless chiral fermions. As will be exhibited, alterations to a medium with the vorticity are twofold; it's required to substitute flat-spacetime derivatives with covariant derivatives that include couplings of the vorticity to the spin. Technically this term is called spin connection. Furthermore, spacial components of the Dirac gamma matrices get boosted along the metric as $\gamma^{i}-\beta^{i} \gamma^{0}$, where we can interpret $\beta^{i}$ as the speed of relative local patches that cover the spacetime. The prescribed modifications are dictated by the covariance of the field theory in curved spacetime and they will serve a significant role in our discussion. As we shall see, momenta of the spinors are subject to a shift by $\pm \lambda \omega^{z}$ with $\lambda=\frac{1}{2}$, depending on whether the spinors are right-handed or left-handed and also the direction of the vorticity. As we will observe, the interpretation employs a coupling between the orbital angular momentum and the spin. In virtue of the covariance of the Dirac equation, dispersion relations acquire a new kinetic term of the form $\beta^{i} p_{i}$, with $p_{i}$ the momenta of the spinors.

Special focus is on chiral fermions. Our goal is to systematically expand the background perturbations to first order and derive the equations of motion and the corresponding solutions of the Dirac equation. Along the way, we study the Hamiltonian and the chiral current from the first principles. This is done in the most convenient way through the second quantization. This will allow us to comprehend the separation of the right-handed 
or left-handed fermions under the influence of an external vorticity microscopically. To embody the later, primarily we study the effects of the chemical potential and the background electromagnetic potentials on interactions that are present in the theory.

In section 2. we give a short but complete review of the formalism for studying fermions in an arbitrary curved background. Starting from the Lagrangian, we derive the equations of motion and the Hamiltonian. Section 3 . will be the applications of the former tools. In 3.2. we shall square the Hamiltonian operator and investigate the interactions that appear and hence, we infer properties of the eigenvectors and eigenvalues of the Dirac equation. In 3.3. we look into the modifications of the boost and rotation operators produced by vorticities. Finally in 3.4. we compute the chiral vortical current. The summary will recap our main ideas and results.

\section{Review of the tools}

Our goal is to find the Hamiltonian and the equations of motion for free massless fermions in a medium with vorticities with the aid of curved-background methods. To this end, we start with a general formalism and then later on we concentrate on the specific background perturbations that produce vorticities. In this section, we present a brief and self-contained review of the prerequisite material based on [47].

\subsection{Local Lorentz frames}

It is convenient to describe fermions in a curved space using local Lorentz frames $\boldsymbol{e}_{\alpha}$, on a space-time manifold $\mathcal{M}$ which meets the condition $\boldsymbol{e}_{\alpha} \cdot \boldsymbol{e}_{\beta}=\eta_{\alpha \beta}$, with $\eta_{\alpha \beta}$ being the 4D Minkowski metric with the signature of -+++ . The inner product on the above manifold is defined with respect to a metric $g_{\mu \nu}$. There, written in components has the form $e_{\alpha \mu} e_{\beta}^{\mu}=\eta_{\alpha \beta}$ that also satisfies $e_{\alpha \mu} e_{\nu}^{\alpha}=g_{\mu \nu}$. Throughout this section we stick to the following notation for the indices; coordinate indices are chosen from the middle of the Greek alphabet and are lowered and raised by the metric $g_{\mu \nu}$ and its inverse $g^{\mu \nu}$, supplemented by the indices from the first of the alphabet which are called frame indices and are handled by the Minkowski metric $\eta_{\alpha \beta}$ and its inverse $\eta^{\alpha \beta}$.

In order to find the covariant derivatives for spinors we need to consider both of the general coordinate and the Lorentz transformations. In the curved background, derivative of a contravariant vector field $\Psi^{\mu}$ takes the from $\Psi_{; \nu}^{\mu}=\Psi_{, \nu}^{\mu}+\Gamma^{\mu}{ }_{\sigma \nu} \Psi^{\sigma}$, with $\Gamma_{\sigma \nu}^{\mu}$ the Riemann connection $\Gamma_{\sigma \nu \mu}=\frac{1}{2}\left(g_{\sigma \nu, \mu}+g_{\sigma \mu, \nu}-g_{\nu \mu, \sigma}\right)$. On the other hand, the same vector field in the local Lorentz frame is given by the contraction of $\Psi^{\alpha}=e_{\mu}^{\alpha} \Psi^{\mu}$. Then, its covariant derivative can equally well be written in this frame as $\Psi_{; \mu}^{\alpha}=\Psi_{\mu}^{\alpha}+\Sigma_{\beta \mu}^{\alpha} \Psi^{\beta}$, where $\Sigma_{\mu}^{\alpha \beta}$ is known as the spin connection. Thus, relating these two derivatives requires the covariant differentiation to act neutral in passing the coordinate indices to the local Lorentz frame indices,

$$
\Psi_{; \mu}^{\alpha}=e_{\nu}^{\alpha} \Psi_{; \mu}^{\nu}=e_{\nu}^{\alpha}\left(\Psi_{, \mu}^{\alpha}+\Gamma^{\nu}{ }_{\sigma \mu} \Psi^{\sigma}\right),
$$

comparison of the two derivatives leads us to

$$
\Sigma_{\mu}^{\alpha \beta}=e^{\alpha \sigma} e^{\beta \nu} \Gamma_{\sigma \nu \mu}-e_{\nu, \mu}^{\alpha} e^{\beta \nu} .
$$


It's also easy to show that $\Sigma_{\mu}^{\alpha \beta}$ is antisymmetric when $\alpha$ and $\beta$ are interchanged. One usually uses the Dirac matrices that satisfy $\left\{\gamma_{\alpha}, \gamma_{\beta}\right\}=2 \eta_{\alpha \beta}$ in the local Lorentz frame where its contravariant vector takes the form $\gamma^{\mu}=\gamma_{\alpha} e^{\alpha \mu}$, with $\left\{\gamma^{\mu}, \gamma^{\nu}\right\}=2 g^{\mu \nu}$. Thereby, the covariant derivative of the fermionic fields in terms of the spin connection reads

$$
\nabla_{\mu} \Psi=\partial_{\mu} \Psi+\frac{1}{2} G_{[\alpha \beta]} \sum_{\mu}^{\alpha \beta} \Psi, \quad \nabla_{\mu} \bar{\Psi}=\partial_{\mu} \bar{\Psi}-\frac{1}{2} \bar{\Psi} G_{[\alpha \beta]} \sum_{\mu}^{\alpha \beta},
$$

with $G_{[\alpha \beta]}=\frac{1}{4}\left[\gamma_{\alpha}, \gamma_{\beta}\right], \bar{\Psi}=\Psi^{\dagger} \eta$ and $\eta=i \gamma^{0}$.

\subsection{The equations of motion}

Now that we have the form of the covariant derivative of the spinors, we can write the density of the Lagrangian for the massless spinors as

$$
\mathcal{L}=-i g^{1 / 2} \bar{\Psi} \gamma^{\mu}\left(\frac{\partial}{\partial x^{\mu}}+\frac{1}{2} G_{[\alpha \beta]} \Sigma_{\mu}^{\alpha \beta}\right) \Psi
$$

For future advantageous, we will rewrite the above Lagrangian density using the Leibniz's rule,

$$
g^{1 / 2} \nabla_{\mu} \bar{\Psi} \gamma^{\mu} \Psi+g^{1 / 2} \bar{\Psi} \gamma^{\mu} \nabla_{\mu} \Psi=\nabla_{\mu}\left(g^{1 / 2} \bar{\Psi} \gamma^{\mu} \Psi\right)
$$

that follows from the simple identity $\left[\gamma_{\alpha}, G_{[\beta \gamma]}\right] \Sigma^{\beta \gamma}{ }_{\mu}=2 \Sigma_{\alpha}^{\gamma}{ }_{\mu} \gamma_{\gamma}$. In view of the right-hand side of eq. (2.5) that enjoys $\nabla_{\mu}\left(g^{1 / 2} \bar{\Psi} \gamma^{\mu} \Psi\right)=\partial_{\mu}\left(g^{1 / 2} \bar{\Psi} \gamma^{\mu} \Psi\right)$, we can compute both sides separately to arrive at

$$
\bar{\Psi} \partial_{\mu} \gamma^{\mu} \Psi=\frac{1}{2} \bar{\Psi}\left[\gamma^{\mu}, G_{[\alpha \beta]}\right] \Sigma_{\mu}^{\alpha \beta} \Psi .
$$

The Dirac Lagrangian in eq. (2.8) now, takes the form

$$
\mathcal{L}=-i g^{1 / 2} \bar{\Psi}\left[\frac{1}{2}\left\{\gamma^{\mu}, \frac{\partial}{\partial x^{\mu}}\right\}+\frac{1}{4}\left\{\gamma^{\mu}, G_{[\alpha \beta]}\right\} \Sigma_{\mu}^{\alpha \beta}\right] \Psi
$$

and similarly the equations of motion in curved background will be

$$
\left[\frac{1}{2}\left\{\gamma^{\mu}, \frac{\partial}{\partial x^{\mu}}\right\}+\frac{1}{4}\left\{\gamma^{\mu}, G_{[\alpha, \beta]}\right\} \Sigma_{\mu}^{\alpha \beta}\right] \Psi=0 .
$$

At this point, it's simpler to decompose the metric in the following way

$$
g_{\mu \nu}=\left(\begin{array}{cc}
-\alpha^{2}+\beta_{k} \beta^{k} & \beta_{j} \\
\beta_{i} & \eta_{i j}
\end{array}\right), \quad g^{\mu \nu}=\left(\begin{array}{cc}
-\alpha^{-2} & \alpha^{-2} \beta^{j} \\
\alpha^{-2} \beta^{i} & \eta_{i j}-\alpha^{-2} \beta^{i} \beta^{j}
\end{array}\right),
$$

where in the above matrices we used $\beta^{i}=\eta^{i j} \beta_{j}, g=\alpha^{2}$ and $i, j, k \in\{1,2,3\}$. Here $\alpha$ is a positive function of the coordinates $x^{i}$ known as the lapse function and $\beta^{i}$ is called shift vector.

When spacetime is flat, the easiest choice for the components of the local Lorentz frames is simply given by $e_{\alpha \mu}=\eta_{\alpha \mu}$. To adopt a decomposition in curved space, we specialize our study to stationary backgrounds. But prior to that, let's indicate what we mean by the stationary backgrounds. When the metric $g_{\mu \nu}$ and thus the dynamics of the 


\begin{tabular}{|c|c|c|}
\hline Vierbein's components & Time-like coordinate dir. & Space-like coordinate dir. \\
\hline Time-like Lorentz dir. & $e_{0}^{0}=\alpha^{-1}$ & $e_{0}{ }^{i}=-\alpha^{-1} \beta^{i}$ \\
& $e_{00}=-\alpha$ & $e_{0 i}=0$ \\
\hline Space-like Lorentz dir. & $e_{a}^{0}=0$ & $e_{a}{ }^{i} e_{b i}=\eta_{a b}$ \\
& $e_{a 0}=\beta_{i} e_{a}{ }^{i}$ & $e_{a i} e^{a}{ }_{j}=\eta_{i j}$ \\
\hline
\end{tabular}

Table 1. Decomposition of local Lorentz frames.

spinors are independent of time together with the condition $\left|\beta^{i}\right|<1$, the Killing vector associated with the time translation will be timelike. This is the main assumption of this paper.

The fact that the time direction is the Killing vector makes it possible to define local orthonormal frames $\boldsymbol{e}_{a}, a=1,2,3$ on each patch of the $3 \mathrm{D}$ spatial section $x^{0}=t=$ constant, that is $e_{a}^{i} e_{b i}=\eta_{a b}$ and $e_{a i} e^{a}{ }_{j}=\eta_{i j}$, where $a, b \in\{1,2,3\}$.

One then drags each field $\mathbf{e}_{a}$ by the vector $\partial / \partial x^{0}$, to extend the previous orthonormal construction to $4 \mathrm{D}$. All we need to add is $e_{a}^{0}=0$ and $e_{a 0}=\beta_{i} e_{a}^{i}$ and an extra vector field $\mathbf{e}_{0}$ with components $e_{0}^{0}=\alpha^{-1}$ and $e_{0}^{i}=-\alpha^{-1} \beta^{i}$ in addition to $e_{00}=-\alpha$ and $e_{0 i}=0$. We summarize this foliation of spacetime in table 1.

The Hamiltonian is calculated using the usual definition of $H$ as the Legendre transformation of $\mathcal{L}$. This operation yields

$$
H=\int d^{3} \boldsymbol{x} \alpha \bar{\Psi}\left[\frac{1}{2}\left\{\gamma^{i}-\alpha^{-1} \beta^{i} \gamma^{0}, \frac{\partial}{\partial x^{i}}\right\}+\frac{1}{4}\left\{\gamma^{\mu}, G_{[\alpha \beta]}\right\} \Sigma_{\mu}^{\alpha \beta}\right] \Psi
$$

here $\gamma^{\mu}=\gamma^{\alpha} e_{\alpha}^{\mu}$ and $\gamma^{0}$ has the frame index. ${ }^{1}$ To write the first therm in the above bracket, we used table 1. It worth pointing out that this Hamiltonian is exact. In the next section, we shall narrow our study and only consider specific choices of the metric that meets $\Sigma_{0}^{x y} \neq 0$. In addition, we will be interested to know the effect of chemical potentials.

\section{Applications}

\subsection{Vortical dipole moment}

In the previous section, we mentioned the exact Hamiltonian for massless free spinors in curved space. Since we are interested in first order perturbations of the background metric, we simplify eq. (2.10) with the metric given in eq. (2.9) and assume $\partial_{i} \beta^{i}=0$ to deduce

$$
H=\int d^{3} \boldsymbol{x} \bar{\Psi}\left[\left(-\alpha^{-1} \beta^{i} \gamma^{0}+\gamma^{i}\right) \frac{\partial}{\partial x^{i}}+\frac{1}{4}\left\{\gamma^{\mu}, G_{[\alpha \beta]}\right\} \Sigma_{\mu}^{\alpha \beta}+i \gamma^{0} \mu\right] \Psi+\mathcal{O}\left(\alpha^{2}, \beta^{2}\right),
$$

\footnotetext{
${ }^{1}$ We use

$$
\gamma^{0}=\left(\begin{array}{cc}
0 & -i \\
-i & 0
\end{array}\right), \quad \gamma^{a}=\left(\begin{array}{cc}
0 & -i \sigma^{a} \\
i \sigma^{a} & 0
\end{array}\right) \quad \text { with } \quad \gamma^{5}=\left(\begin{array}{cc}
I & 0 \\
0 & -I
\end{array}\right)
$$
}


Let's pause for a moment and compare the Hamiltonian in eq. (3.1) with what we already know from the flat spacetime. As one can notice, adding chemical potential $\mu$ can be confusing; it couples to the conserved charge and the fact that whether we need to write it in terms of $\gamma^{0}$ or $\gamma_{0}$ depends on the normal vector to the equal-time surfaces. We come back to this point and explain more when we want to solve the Dirac equation. In view of the flat space, $\mathcal{H}^{2}$ (the operator form of $H^{2}$ ) has less information and simpler form than the Dirac Hamiltonianin by comparison. We are curious to see a similar expression for $\mathcal{H}^{2}$ in curved space. In the former background metric we have (with $\alpha=1$ ),

$$
\mathcal{H}^{2}=\eta^{-1}\left(\gamma^{i} \frac{\partial}{\partial x^{i}}+\frac{\gamma^{\mu}}{2} G_{[\alpha \beta]} \Sigma_{\mu}^{\alpha \beta}+i \mu \gamma^{0}\right) \eta\left(\gamma^{j} \frac{\partial}{\partial x_{j}}+\frac{\gamma^{\nu}}{2} G_{[\alpha \beta]} \Sigma_{\nu}^{\alpha \beta}+i \mu \gamma^{0}\right),
$$

where here none of the gamma matrices have the Lorentz index (i.e. frame index). The expansion of the product gives

$$
\begin{aligned}
\mathcal{H}^{2}= & \eta^{-1} \gamma^{i} \eta \gamma^{j} \nabla_{i} \nabla_{j}+\frac{1}{2} \eta^{-1} \gamma^{i} \eta \gamma^{0} G_{[\alpha \beta]} \Sigma_{0}^{\alpha \beta} \nabla_{i}+\frac{1}{2} \eta^{-1} \gamma^{0} G_{[\alpha \beta]} \eta \Sigma_{0}^{\alpha \beta} \gamma^{j} \nabla_{j}+i \mu \eta^{-1} \gamma^{i} \eta \gamma^{0} \nabla_{i} \\
& +i \mu \eta^{-1} \gamma^{0} \eta \gamma^{j} \nabla_{j}+\frac{i}{2} \mu \eta^{-1} \gamma^{0} \eta \gamma^{0} G_{[\alpha \beta]} \Sigma_{0}^{\alpha \beta}+\frac{i}{2} \mu \eta^{-1} \gamma^{0} G_{[\alpha \beta]} \eta \gamma^{0} \Sigma_{0}^{\alpha \beta}-\mu^{2}
\end{aligned}
$$

in arriving at the later form, we have separated the spacial components of $\gamma^{\mu}$ in the second terms of the parentheses in eq. (3.2) and combined them with partial derivatives to construct the spacial components of the covariant derivatives. As we mentioned in the introduction, consider a situation with the constant vorticities parallel to the $z$-axis, then one configuration would be provided by $\beta^{x}(y), \beta^{y}(x) \neq 0$. One can check that the non-zero components of the spin connection are then given by

$$
\begin{aligned}
& \Sigma^{0 y}{ }_{x}=-\frac{1}{2}\left(\partial_{x} \beta^{y}+\partial_{y} \beta^{x}\right), \\
& \Sigma_{y}^{0 x}=-\frac{1}{2}\left(\partial_{x} \beta^{y}+\partial_{y} \beta^{x}\right), \\
& \Sigma_{0}^{x y}=\frac{1}{2}\left(\partial_{y} \beta^{x}-\partial_{x} \beta^{y}\right) .
\end{aligned}
$$

If we define $\omega^{z} \equiv-\frac{1}{2} \partial_{y} \beta_{x}$ and assume a symmetric setup that has the property of $-\partial_{x} \beta^{y}=$ $\partial_{y} \beta^{x}$, then the above expressions reduce to $\Sigma^{0 y}{ }_{x}=0, \Sigma^{0 x}{ }_{y}=0$ and $\Sigma^{x y}{ }_{0}=-2 \omega_{z}$. The prefactor of two in the later result can be interpreted as having double vorticities in the same direction.

With the utility of $\nabla_{i} \nabla_{j}-\nabla_{j} \nabla_{i}=-\frac{1}{2} G_{[\alpha \beta]} R^{\alpha \beta}{ }_{i j}$, we shall symmetrize the curved space gradients in eq. (3.1) for $R_{k l}^{0 j}=\partial_{j} \partial_{[k} \beta_{l]}$ vanishes in the limit of constant vorticities. After carrying out necessary algebraic rearrangements, we infer that

$$
\begin{aligned}
H^{2}=\int d^{3} \boldsymbol{x} \bar{\Psi}\left[\left(P_{x}-2 i \omega_{z} \gamma^{y} \gamma^{0}\right)^{2}+\left(P_{y}+2 i \omega_{z} \gamma^{x} \gamma^{0}\right)^{2}+P_{z}^{2}\right. \\
\left.+4\left(\beta^{x} P_{x}+\beta^{y} P_{y}-\mu\right) G^{[0 a]} P_{a}-2 \mu \omega_{z} \sigma^{z}-\mu^{2}\right] \Psi,
\end{aligned}
$$

where $G^{[0 a]}=\frac{1}{4}\left[\gamma^{0}, \gamma^{a}\right]$ and $P_{i}=-i \partial_{i}$. As we will discuss more carefully in the next section, the energy eigenvalues are shifted by $\beta^{i} P_{i}$, therefore naturally cross terms with $\beta^{i}$ 
appear in the eigenvalues of $H^{2}$. The term proportional to $\sigma^{z}$ in eq. (3.5) is understood better in the Weyl representation

$$
-\mu 2 \omega^{z}\left(\Psi_{R}^{\dagger} \Psi_{L}-\Psi_{L}^{\dagger} \Psi_{R}\right)
$$

This term, $\boldsymbol{\mu} \boldsymbol{\omega} \cdot \boldsymbol{\sigma}$, is identical to the magnetic dipole moment in the $\boldsymbol{\mu}_{B} \cdot \boldsymbol{B}$ with $\boldsymbol{\mu}_{B}=\frac{\boldsymbol{\sigma}}{2}$ for spin- $\frac{1}{2}$ fermions in an external background magnetic field. In this case vortical dipole moment is defined by $\mu_{V}=\mu \boldsymbol{\sigma}$.

For the case that $\mu=0$, we can understand the shifts in the momenta $P_{x}$ and $P_{y}$. The $z$ component of the total angular momentum, $\boldsymbol{J}=\boldsymbol{L}+\boldsymbol{S}$, commutes with $H^{2}$ whence $J_{z}$ is conserved and its eigenvalues, $m$, can be used for labeling the states of the system i.e. $J_{z}|\Psi\rangle=m|\Psi\rangle$.

Recalling the configuration in eq. (3.4), we can think of a setup that constitutes two vorticities parallel but in the opposite directions. This is achieved by the condition $\partial_{x} \beta^{y}=$ $\partial_{y} \beta^{x}$. Thus, the squared of the Hamiltonian will take the form

$$
\begin{aligned}
H^{2}=\int d^{3} \boldsymbol{x} \bar{\Psi}\left[\left(P_{x}-2 i \omega_{z} \gamma^{y} \gamma^{0}\right)^{2}+\left(P_{y}-2 i \omega_{z} \gamma^{x} \gamma^{0}\right)^{2}+P_{z}^{2}\right. \\
\left.+4\left(\beta^{x} P_{x}+\beta^{y} P_{y}-\mu\right) G^{[0 a]} P_{a}-\mu^{2}\right] \Psi,
\end{aligned}
$$

which means that the operator $J_{z}$ does not commute with the squared of the Hamiltonian and in addition, the vortical dipole term is absent.

\subsection{Dirac equation}

The expression for the Dirac equation in a general curved background was already mentioned in eq. (2.8). To consider the general case, we also need to add a chemical potential to the equation. This requires understanding of time-like vectors in our metric

$$
d s^{2}=-d t^{2}+\left(d x+\beta^{x}(y) d t\right)^{2}+d y^{2}+d z^{2},
$$

with $\beta^{x}(y)$ some arbitrary function of $y$. Note that in the rest of our discussion, we will consider a simpler setup than what we used in the last section which assumes $\beta^{x}(y) \neq 0$ but $\beta^{y}(x)=0$. Different configurations of this sort just change the prefactor of $\omega^{z}$ in the equations. The metric in eq. (3.8) is a rectilinear model of the Kerr metric and has interesting properties [48]; the hypersurfaces of constant $t$ are spacelike and they satisfy Cauchy problem. In addition, the metric and the dynamics of fields are also time independent. The stationary condition can be violated only if $\left|\beta^{x}(y)\right| \geq 1$ for some $y$. It's the result of the time independence of the problem that the norm of the normal vector to the equal-time hypersurfaces, $\partial_{\mu} t=(1,0,0,0)$, is greater than zero and time is an ignorable coordinate. We keep in mind that, contravariant components of the Killing vector responsible for time translations are $\partial_{t} x^{\mu}=(1,0,0,0)$ with $g_{00}=-1+\beta_{x}^{2}$. Therefore, when the condition $\left|\beta^{x}\right|>1$ is satisfied, the Killing vector is spacelike and the metric is no longer stationary. But in this paper, we keep curved space corrections upto $\mathcal{O}\left(\beta_{x}^{2}\right)$ so our configuration is manifestly time-independent and stationary. 


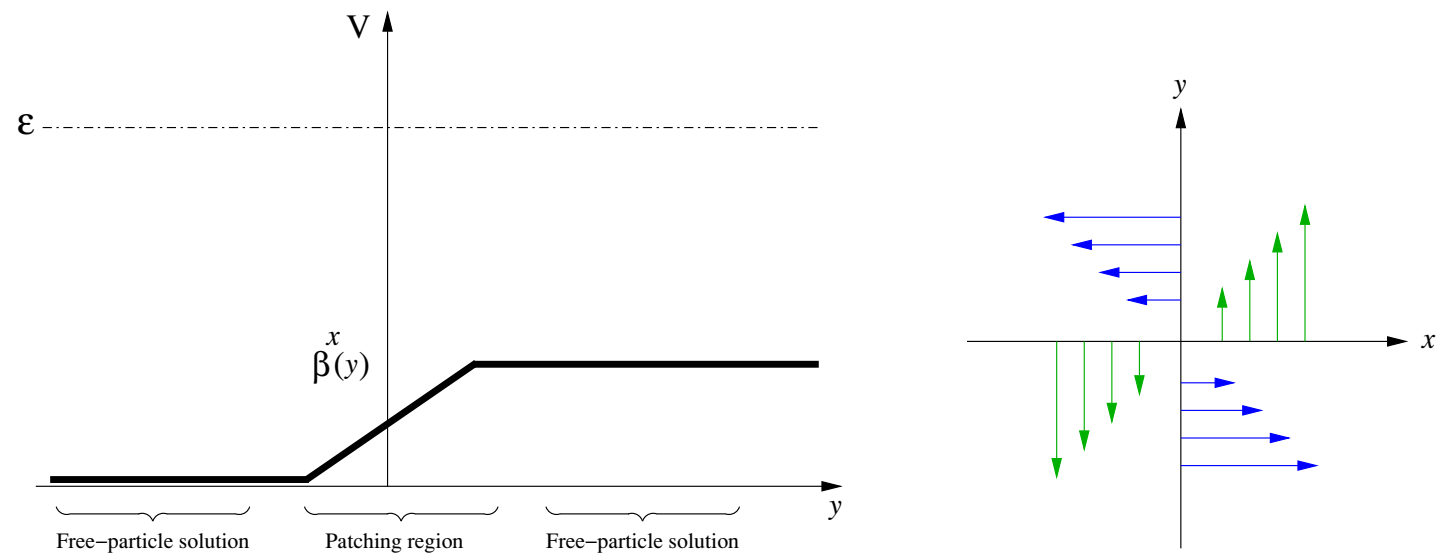

Figure 1. (Left side) Dirac equation in the curved background is illustrated by spinors in a potential well. Throughout our calculation we will be working in the semiclassical regime that WKB approximation is valid. (Right side) Picture of a rotating fluid made of $\beta^{x}(y)$ and $\beta^{y}(x)$ profiles in blue and green colors respectively.

We seek a solution of $\Psi(t)=\chi e^{-i \varepsilon t}$ for $\varepsilon>0$. Adding chemical potential, $-i \mu \gamma^{0}$ to the equations of motion, it is understood that

$$
\left(-i \varepsilon \gamma^{0}-i \mu \gamma^{0}+\frac{1}{2}\left\{\gamma^{i}, \frac{\partial}{\partial x^{i}}\right\}+\frac{1}{4}\left\{\gamma^{\mu}, G_{[\alpha \beta]}\right\} \Sigma_{\mu}^{\alpha \beta}\right) \chi=0 .
$$

Simplification of the above equation gives

$$
\left(\begin{array}{cc}
0 & -i(\varepsilon+\mu)-\beta^{x} \partial_{x}+\sigma^{i} \partial_{x^{i}}-i \lambda \omega^{z} \sigma^{z} \\
-i(\varepsilon+\mu)-\beta^{x} \partial_{x}-\sigma^{i} \partial_{x^{i}}-i \lambda \omega^{z} \sigma^{z} & 0
\end{array}\right) \chi=0,
$$

with $\lambda=\frac{1}{2}$. As we mentioned earlier, in general $\beta^{x}(y)$ is an arbitrary function of the $y$ coordinate. Requiring vorticity to be constant means that $\beta^{x}(y)$ has to be a linear function and in principle one has to solve the Dirac equation in this external potential. However, we are interested at a regime ${ }^{2}$ that $\varepsilon \gg \beta^{x}(y)$. Then normalizations of waves are not affected by this background and we can neglect the reflection of the waves from the potential.

On both sides of the potential well, solutions of the Dirac equation are those of the free fermions except that on the right-hand side, energy eigenvalues are shifted by the constant value of $\beta^{x} P_{x}$. Furthermore, eq. (3.10) shows that transition from the left to the right region is accompanied by a discontinuity in the momentum $P^{z}$ by $\lambda \omega^{z}$. Another way of saying this is that we use WKB approximation for the mid region. Then the solutions of this problem are simply obtained by the following replacements

$$
\begin{array}{lll}
u_{R}: & p^{0} \rightarrow p^{0}+\mu+\beta^{x} p_{x}, & p_{z} \rightarrow p_{z}-\lambda \omega^{z}, \\
u_{L}: & p^{0} \rightarrow p^{0}+\mu+\beta^{x} p_{x}, & p_{z} \rightarrow p_{z}+\lambda \omega^{z},
\end{array}
$$

in the solutions for the right-handed and left handed free spinors.

\footnotetext{
${ }^{2}$ We make this choice to neglect the effect of the superradiant modes i.e. reflected waves from a potential barrier.
} 
The fact that energy eigenvalues depend on $\beta^{x}$ bears more attention. One property of the metric in eq. (3.8) is that under the Galilean coordinate transformation of $x^{\prime}=x-\beta t$ with $t^{\prime}=t, y^{\prime}=y$ and $z^{\prime}=z$, we will have a gauge transformation in the form $\beta_{x}^{\prime}(y) \equiv$ $\beta_{x}(y)+\beta$. This means that frequency and energy are determined up to $\beta P^{x}$. As it has been pointed out by Fulling [48], the energy difference between two states with different $P^{x}$ is ill-defined, but the conservation of momentum in $x$ direction that is preserved by the Killing vector $\partial_{x}$, forbids such transitions. The energies

$$
\begin{aligned}
& \varepsilon_{R}+\mu+\beta^{x} p_{x}= \pm|\boldsymbol{p}| \mp \lambda \frac{p_{z}}{|\boldsymbol{p}|} \omega^{z}, \\
& \varepsilon_{L}+\mu+\beta^{x} p_{x}= \pm|\boldsymbol{p}| \pm \lambda \frac{p_{z}}{|\boldsymbol{p}|} \omega^{z}
\end{aligned}
$$

for the right-handed and left-handed spinors, are advantageously presented for the future calculations. There, upper and lower signs are for particles and antiparticles respectively.

Since we are going to adopt the second-quantization approach in the next section, we shall elaborate on the role played by the creation and annihilation operators. Essentially, they should be consistent with the above pattern for energies. For instance, in terms of the expansion over momentum $\boldsymbol{p}$, operators $a_{\boldsymbol{p}}^{\dagger}$ and $a_{\boldsymbol{p}}$ create and annihilate particles with the momenta $p_{z} \pm \lambda \omega^{z}$ and the energies given in eq. (3.13). To keep the discussion as simple as possible we only consider the right-handed particles.

\subsection{Modified Lorentz generators}

In this section, we study the effect of vorticities on Lorentz generators. Since chemical potentials explicitly break the Lorentz invariance we assume that $\mu=0$ in the following section.

In an arbitrary background, Lorentz generators of boosts and rotations, do not satisfy their flat spacetime commutation relations of $\mathrm{SO}(3,1)$ globally,

$$
\left[M_{\mu \nu}, M_{\sigma, \tau}\right]=\eta_{\mu \sigma} M_{\nu \tau}+\eta_{\nu \tau} M_{\mu \sigma}-\eta_{\mu \tau} M_{\nu \sigma}-\eta_{\nu \sigma} M_{\mu \tau}
$$

with

$$
M_{\alpha \beta}=-\int_{\Sigma} d \Sigma_{\rho} \mathcal{M}_{\alpha \beta}{ }^{\rho},
$$

here $\Sigma$ is any spacelike Cauchy hypersurface with the differential element of $d \Sigma_{\rho} \equiv d \Sigma n_{\rho}$. As explained before, surfaces of constant $t$ are spacelike and the normal vectors to the equal-time surfaces are given by $n_{\rho}=\partial_{\rho} t=(1,0,0,0)$. The generalized angular momentum density, $\mathcal{M}_{\alpha \beta}{ }^{\rho}$ is defined [49-51] by

$$
\mathcal{M}_{\alpha \beta}{ }^{\rho}=-\left(x_{\alpha} T_{\beta}{ }^{\rho}-x_{\beta} T_{\alpha}{ }^{\rho}\right)
$$

with

$$
T^{\rho \alpha}=-\frac{i g^{1 / 2}}{4}\left(\nabla^{\rho} \bar{\Psi} \gamma^{\alpha} \Psi+\nabla^{\alpha} \bar{\Psi} \gamma^{\rho} \Psi-\bar{\Psi} \gamma^{\rho} \nabla^{\alpha} \Psi-\bar{\Psi} \gamma^{\alpha} \nabla^{\rho} \Psi\right)
$$

here $T^{\mu \nu}$ is the stress tensor density defined by $T^{\mu \nu}=\frac{\delta S}{\delta e_{\alpha \mu}} e_{\alpha}^{\nu}$ with the action given in eq. (2.4). This is the equivalent definition of the energy-momentum tensor density $T^{\mu \nu}=$ 
$2 \frac{\delta S}{\delta g_{\mu \nu}}$ for fermionic fields in curved spacetime. In eq. (3.17), covariant derivatives are defined in eq. (2.3). The terms in eq. (3.16) comprise both the orbital angular momentum and the spin. In the flat spacetime, ${ }^{3}$ these generators are known for free spinors $\left(p^{0}=|\boldsymbol{p}|\right)$

$$
M_{\alpha \beta}=\frac{i}{2} \int_{\boldsymbol{p}} a_{\boldsymbol{p}}^{\dagger} u_{R}^{\dagger}(\boldsymbol{p})\left[L_{\alpha \beta}(\boldsymbol{p})-2 G_{[\alpha \beta]}\right] u_{R}(\boldsymbol{p}) a_{\boldsymbol{p}}
$$

where here

$$
L_{\alpha \beta}(\boldsymbol{p})=\overleftrightarrow{p_{\alpha} \frac{\partial}{\partial p^{\beta}}}-\overleftrightarrow{p_{\beta} \frac{\partial}{\partial p^{\alpha}}},
$$

and by $\leftrightarrow$ on top of an operator, we mean the antisymmetric action on the right and left side of the operator. The coefficients in the expansion are defined based on the creation and the annihilation operators for the right-handed fermions. As we discussed in the previous section, we consider solutions for spinors that are almost those of the flat background except that in a mid transition region, energy eigenvalues are slowly varying functions of $\beta^{x}(y)$. This will also give rise to a nonzero derivative of the potential $\omega^{z} \equiv-\frac{1}{2} \partial_{y} \beta^{x}$.

So how do Lorentz generators look like in this geometry? To answer this question we study the definitions mentioned in eq. (3.16) and eq. (3.17). Our new spinors are similar to the ones in flatspace but momentum arguments are now in terms of the shifted momenta $p_{z}-3 \lambda \omega^{z}$. Another contribution comes from the spin connection in covariant derivatives of eq. (3.17). We look into the generators of boosts and rotations separately.

Boosts. - As a first example, we will look into the boost along the $z$ direction, $M^{0 z}=$ $\int d^{3} \boldsymbol{x}\left(t T^{0 z}-z T^{00}\right)$. Below we study each of these terms using the second-quantized formulation. Although we restrict ourselves to $t=0$, it's instructive to start with $T^{0 z}$. Its component from eq. (3.17) explicitly reads $\left(g^{1 / 2}=-i\right)$,

$$
T^{0 z}=\frac{-1}{4} \bar{\Psi}\left[\gamma^{z}\left(\overleftrightarrow{\partial_{0}}-\beta^{x} \overleftrightarrow{\partial_{x}}\right)-\gamma^{0} \overleftrightarrow{\partial_{z}}+\left\{G_{x y}, \gamma^{z}\right\} \Sigma_{0}^{x y}\right] \Psi
$$

here gamma matrices with the coordinate indices are equal to ones carrying frame indices and we have rewritten the derivatives in terms of their covariant components. Expansion over right-handed spinors, neglecting antiparticles, gives

$$
\frac{1}{2} \int_{\boldsymbol{p}} u_{R}^{\dagger}(\boldsymbol{p}) a_{\boldsymbol{p}}^{\dagger}\left[\sigma^{z}\left(p^{0}+\beta^{x} p_{x}\right)+p^{z}+\lambda \omega^{z}\right] u_{R}(\boldsymbol{p}) a_{\boldsymbol{p}}
$$

in which $\int_{\boldsymbol{p}} \equiv \int \frac{d^{3} p}{(2 \pi)^{3}}$. To take the volume integral, we have assumed that variation of $\beta^{x}(y)$ is ignorable comparing to the oscillatory Fourier factor (WKB approximation). We can compute $u_{R}^{\dagger}(\boldsymbol{p}) \sigma^{z} u_{R}(\boldsymbol{p})$ using the Gordon identity

$$
(|\boldsymbol{p}|+|\boldsymbol{q}|) u_{R}^{\dagger}(\boldsymbol{q}) \sigma^{z} u_{R}(\boldsymbol{p})=u_{R}^{\dagger}(\boldsymbol{q})\left(q^{z}+p^{z}+\frac{1}{2}\left[\sigma^{z}, \sigma^{j}\right]\left(p_{j}-q_{j}\right)\right) u_{R}(\boldsymbol{p}) .
$$

Similar to the above identity, the sandwich of $\sigma^{x}$ and $\sigma^{y}$ is evaluated by replacing $|\boldsymbol{p}|$ and $|\boldsymbol{q}|$ with $|\boldsymbol{p}|-\lambda \omega^{z} \hat{p}_{z}$ and $|\boldsymbol{q}|-\lambda \omega^{z} \hat{q}_{z}$ on the left-hand side accordingly and trivially replacing

\footnotetext{
${ }^{3}$ We restrict our discussion to $t=0$ for simplicity.
} 
the $z$ indices with $x$ and $y$ on the right-hand side. Suitable simplifications of eq. (3.21) enable us to write it in the suggestive form

$$
\int d^{3} \boldsymbol{x} T^{0 z}=\frac{1}{2} \int_{\boldsymbol{p}} u_{R}^{\dagger}(\boldsymbol{p}) u_{R}(\boldsymbol{p})\left[p_{z}+\lambda \omega^{z}-\hat{p}_{z} p_{0}\right] a_{\boldsymbol{p}}^{\dagger} a_{\boldsymbol{p}}
$$

with $-p_{0} \equiv p^{0}+\beta^{x} p_{x}$.

Another non trivial contribution to the boost operator, $M^{0 z}$, comes from the term $\int d^{3} \boldsymbol{x} z T^{00}$ with $T^{00}$ from eq. (3.17) since the spin connection is not zero, $\Sigma_{0}^{x y}=-\omega^{z}$, as it's clear from eq. (3.20) by replacing $z$ with 0 coordinate. After Fourier expansion and decomposing different modes, the integral reads ${ }^{4}$

$$
\int d^{3} \boldsymbol{x} z T^{00}=\frac{1}{2} \int d^{3} \boldsymbol{x} z \int_{\boldsymbol{p}, \boldsymbol{q}} a_{\boldsymbol{q}}^{\dagger} u_{R}^{\dagger}(\boldsymbol{q})\left[-p_{0}-q_{0}+\omega^{z}\left\{\gamma^{0}, G_{[x y]}\right\}\right] u_{R}(\boldsymbol{p}) a_{\boldsymbol{p}} e^{i \boldsymbol{x} \cdot(\boldsymbol{p}-\boldsymbol{q})} .
$$

Applying $\left\{\gamma^{0}, G_{[x y]}\right\}=i \sigma^{z} \gamma^{0}$ and use of the following identity,

$$
\int d^{3} \boldsymbol{x} x^{j} \int_{\boldsymbol{p}, \boldsymbol{q}} e^{i \boldsymbol{x} \cdot(\boldsymbol{p}-\boldsymbol{q})} f(\boldsymbol{p}, \boldsymbol{q})=\frac{i}{2} \int_{\boldsymbol{p}, \boldsymbol{q}} \delta^{3}(\boldsymbol{p}-\boldsymbol{q})\left(\frac{\partial}{\partial p_{j}}-\frac{\partial}{\partial q_{j}}\right) f(\boldsymbol{p}, \boldsymbol{q}),
$$

yields

$$
\int d^{3} \boldsymbol{x} z T^{00}=-\frac{i}{2} \int_{\boldsymbol{p}} a_{\boldsymbol{p}}^{\dagger} u_{R}^{\dagger}(\boldsymbol{p})\left[\overleftrightarrow{p_{0} \frac{\partial}{\partial p_{z}}}+\frac{\omega^{z} \sigma^{z}}{4} \frac{\vec{\partial}}{\partial p_{z}}\right] u_{R}(\boldsymbol{p}) a_{\boldsymbol{p}}
$$

Our argument in this calculation was general and we could generalize it to $M^{0 x}$ and $M^{0 y}$,

$$
M^{0 i}=\frac{i}{2} \int_{\boldsymbol{p}} a_{\boldsymbol{p}}^{\dagger} u_{R}^{\dagger}(\boldsymbol{p})\left[\stackrel{\overrightarrow{p_{0}} \frac{\partial}{\partial p_{i}}}{+}+\frac{\omega^{z} \sigma^{z}}{4} \frac{\vec{\partial}}{\partial p_{i}}\right] u_{R}(\boldsymbol{p}) a_{\boldsymbol{p}}
$$

and in the above, the first term has the form similar to $M_{\text {flat }}^{0 i}$ in eq. (3.18) which is the contribution in the absence of the vorticities.

Rotations. It's also interesting to extend our computation to generators of rotations. The simplest case is $M^{x y}$, rotations around the vorticity. As we have seen in section 3 the component of the angular momentum along the direction of vorticities commutes with the Hamiltonian. Simple calculation here also confirms that this generator is not modified by vorticity corrections $M^{x y} \equiv M_{\text {flat }}^{x y}$. But this is certainly not true for $M^{x z}$ and $M^{y z}$. Similar computation gives

$$
M^{x z}=M_{\text {flat }}^{x z}+\frac{i \omega^{z}}{8} \int_{\boldsymbol{p}} a_{\boldsymbol{p}}^{\dagger} u_{R}^{\dagger}(\boldsymbol{p})\left[\left(1-\hat{p}_{z}^{2}\right) \frac{\overleftrightarrow{\partial}}{\partial p_{x}}+\frac{2 \hat{p}_{z}}{|\boldsymbol{p}|} G_{[x z]}\right] u_{R}(\boldsymbol{p}) a_{\boldsymbol{p}}
$$

with the identical expression ${ }^{5}$ for $M^{y z}$ by a simple interchange of $x \leftrightarrow y$.

\footnotetext{
${ }^{4}$ To avoid defining new notations, we will constantly use $G_{[\alpha \beta]}$ sandwiched by the right-handed spinors, in spite of the fact that they are doublets.

${ }^{5}$ Also note that by $M_{\text {flat }}^{x y}$ or $M_{\text {flat }}^{x z}$, we mean the their forms are identical to their corresponding flatspacetime counterparts, otherwise their expansions are in terms of the creation and annihilation operators prescribed below eq. (3.13).
} 
We would like to give emphasis to the role played by $T^{0 z}$ in the structure of the boosts and rotations. Since this component of the stress tensor has an extra contribution proportional to the vorticity, commutation relations will be modified. We postpone further study of this issue to future works.

\subsection{Chiral vortical effect}

So far we have covered different aspects of the fermionic theories in the presence of the background vorticity. One novel aspect in this regard is the chiral vortical effect. The electric current density deduced from the variation of the action in eq. (2.4),

$$
\mathcal{L}=-i g^{1 / 2} \bar{\Psi} \gamma^{\mu}\left(\frac{\partial}{\partial x^{\mu}}-i e A_{\mu}+\frac{1}{2} G_{[\alpha \beta]} \Sigma_{\mu}^{\alpha \beta}\right) \Psi
$$

with respect to the electromagnetic four-potential $A_{\mu}(x)$ is $j^{\mu}=-g^{1 / 2} e \bar{\Psi} \gamma^{\mu} \Psi$. Specifically the $z$ component, the direction along the vorticity, of the total current for the right-handed fermions reads

$$
J^{z}=e \int d^{3} \boldsymbol{x} \int_{\boldsymbol{p}, \boldsymbol{q}}\left[u_{R}^{\dagger}(\boldsymbol{q}) \sigma^{z} u_{R}(\boldsymbol{p}) a_{\boldsymbol{q}}^{\dagger} a_{\boldsymbol{p}}\right] e^{i \boldsymbol{x} \cdot(\boldsymbol{p}-\boldsymbol{q})} .
$$

We can use the Gordon's identity in eq. (3.22) to simplify the above expression. Successive substitutions indicate that

$$
J^{z}=e \int d^{3} \boldsymbol{x} \int_{\boldsymbol{p}, \boldsymbol{q}} u_{R}^{\dagger}(\boldsymbol{q}) a_{\boldsymbol{q}}^{\dagger}\left[\frac{p^{z}+q^{z}}{|\boldsymbol{p}|+|\boldsymbol{q}|}-i \frac{p^{x}+q^{x}}{|\boldsymbol{p}|+|\boldsymbol{q}|} \frac{p^{y}-q^{y}}{|\boldsymbol{p}|+|\boldsymbol{q}|}\right] u_{R}(\boldsymbol{p}) a_{\boldsymbol{p}} e^{i \boldsymbol{x} \cdot(\boldsymbol{p}-\boldsymbol{q})},
$$

with corrections of $\mathcal{O}\left(\Delta p^{2}\right)$ with $\Delta p \equiv p-q$ that are suppressed. To establish a connection with CVE, we compute the thermodynamic average by

$$
\left\langle J^{z}\right\rangle=\frac{\sum_{\alpha} \exp \left\{-\frac{\varepsilon_{\alpha}}{T}\right\}\left\langle\alpha\left|J^{z}\right| \alpha\right\rangle}{\sum_{\alpha} \exp \left\{-\frac{\varepsilon_{\alpha}}{T}\right\}},
$$

where each state is marked with a label $\alpha$ and the corresponding energy is $\varepsilon_{\alpha}$. In the absence of the vorticities, this gives $\left\langle a_{\boldsymbol{q}}^{\dagger} a_{\boldsymbol{p}}\right\rangle=\delta^{3}(\boldsymbol{q}-\boldsymbol{p}) f(|\boldsymbol{p}|)$ with $f(\omega) \equiv\left(e^{\frac{\omega}{T}}-1\right)^{-1}$. But as intended in section 3, the influence of the vorticities change the dispersion relation and for the distribution function, we have $f\left(|\boldsymbol{p}|-\beta^{x}(y) p_{x}-\lambda \omega^{z} \hat{p}_{z}\right)$. Rewriting $p^{y}-q^{y}$ in the coordinate space and expanding the anisotropic distribution function over its argument, we can express eq. (3.31) as

$$
\left\langle J^{z}\right\rangle=\left.e \int d^{3} \boldsymbol{x} \int_{\boldsymbol{p}, \boldsymbol{q}} u_{R}^{\dagger}(\boldsymbol{q}) u_{R}(\boldsymbol{p}) e^{i \boldsymbol{x} \cdot(\boldsymbol{p}-\boldsymbol{q})} \frac{\partial f}{\partial \varepsilon}\right|_{f=f_{0}} \delta(\boldsymbol{p}-\boldsymbol{q})\left[\frac{p^{z}+q^{z}}{|\boldsymbol{p}|+|\boldsymbol{q}|}-\frac{p^{x}+q^{x}}{(|\boldsymbol{p}|+|\boldsymbol{q}|)^{2}} \frac{\partial}{\partial y}\right] \varepsilon_{R},
$$

where $f_{0}$ displays the equilibrium distribution function. Since $\partial_{y}$ only acts on $\beta^{x}$ giving a factor of $\omega^{z}=-\frac{\partial_{y} \beta^{x}}{2}$ and hence a constant, the volume integral would be trivial. Integration over $\boldsymbol{q}$ then yields

$$
\frac{\left\langle J^{z}\right\rangle}{V}=\left.e \omega^{z} \int_{\boldsymbol{p}} u_{R}^{\dagger}(\boldsymbol{p}) u_{R}(\boldsymbol{p}) \frac{\partial f}{\partial \varepsilon}\right|_{f=f_{0}}\left(-\frac{\hat{p}_{z}^{2}}{2}-\hat{p}_{x}^{2}\right)
$$


with $\int d^{3} \boldsymbol{x}=V$. This is the expression for CVE obtained for a massless right-handed spin- $\frac{1}{2}$ particle. A similar statement applies to the left-handed spin- $\frac{1}{2}$ particles, with an opposite overall sign. A derivation based on rotating frames and phase-space action has appeared recently in $[20,21]$.

\section{Summary}

In the forgoing discussion, we studied the chiral fermions under the influence of the vorticities. Our analysis is intrigued by the recent advances in the theory of hydrodynamics and treatments used in this work is no surprise for experts on the Kerr black hole. Using the standard methods of quantum field theory in curved spacetime, we derive the Lagrangian, Hamiltonian and the equations of motion for the chiral fermions. Tentatively, we constructed $H^{2}$, the squared of the Hamiltonian. This was primarily important since similar operation in the flat spacetime gives the Hamiltonian, corresponding to the Schrödinger equation for fermions, which is devoid of the spin degrees of freedom. In this case, endowed with the vorticities, a similar interaction to the medium under a background magnetic field appears. The chemical potential times the Pauli sigma matrices acts as a dipole moment under the vorticities. It is also interesting to observe that having two vorticities in opposite directions do not totally degrade or neutralize each other. Some specific interactions that appear in $H^{2}$ are due to moving frames and independent of the former directions.

Rather than looking for less soluble models, we have pursued the Dirac equation for energies $\varepsilon \gg \beta^{x}(y)$. This allows us to deploy the WKB approximation to solve for the solutions in this background that brought a firmer physical interpretation. For instance, for the right-handed spinors, substitution of $p^{0} \rightarrow p^{0}+\mu+\beta^{x} p_{x}$ and $p_{z} \rightarrow p_{z}-\lambda \omega^{z}$, with $\lambda=\frac{1}{2}$ manifests the solution for the right-handed spinors under the action of the vorticities. The new dispersion relation for $\varepsilon_{R}$ agrees with [20,21] while those authors reasoned based on rotating frames. We point out again that as far as we are in the regime of the validity of the WKB approximation, $\beta^{x}(y)$ can have any arbitrary shape.

Another invoking argument was based on the generators of boosts and rotations under the ambient vorticities. Our computation is a prototype of its kind in this context. Here there has been a fundamental assumption about the configurations that we pick up. We only consider the static and stationary backgrounds. Hence, we can use the second quantization. Since the above mentioned operators are defined based on stress tensors, one is curious about the role that $T^{0 z}$ plays while having $\omega^{z} \neq 0$. Direct calculation shows that there are two contributions coming from this term. One is due to the fact that $p^{z}$ has a shift proportional to the vorticity and in addition the spin connection $\Sigma^{x y}{ }_{0}$ is not zero. The final conclusion will be that all generators comprised of $T^{0 z}$, are subject to corrections proportional to the vorticity to first order.

A fascinating continuing body of work has been invested on the topic of the chiral vortical effects in recent years. Our argument is intimately tied to the rudimentary form of the Lagrangian. One important step is to pay attention to the creation and annihilation operators in the background of the vorticities, since these operators now create and annihilate particles out of the vacuum with shifted energies and momenta. This is signified 
in the average ensemble and the distribution function that thereby will be a function of $f\left(|\boldsymbol{p}|-\beta^{x}(y) p_{x}-\lambda \omega^{z} \hat{p}_{z}\right)$. The first term is of course the energy of the massless spinors in the flat spacetime. The second and third terms, $\boldsymbol{u} \cdot \boldsymbol{p}$ and $\lambda \boldsymbol{\omega} \cdot \hat{p}$, are corrections to the energy of an observer in a rotating frame.

In short, it may well be that similar to the case of the electromagnetism that implementation of the electromagnetic four-potentials admits one to study such systems, methods in curved spacetime are the proper way of probing relativistic field theories under the influence of the vorticities.

To close the discussion, we point out again that in this paper small and constant vorticities are considered. Extensions to more general cases are accompanied by the inclusion of superradiant and Klein modes. It is also very important to make a connection to non-equilibrium physics such as turbulence [52], for a better understanding of the holographic picture.

\section{Acknowledgments}

I would like to thank Dam T. Son and Mikhail A. Stephanov for discussion when I began this work. Discussions with Matthias Blau and Adolfo Guarino were also of help during the course of this work. I would also like to thank Mikko Laine and Guy D. Moore for their support. This work was partly supported by the Swiss National Science Foundation (SNF) under grant 200021-140234.

Open Access. This article is distributed under the terms of the Creative Commons Attribution License (CC-BY 4.0), which permits any use, distribution and reproduction in any medium, provided the original author(s) and source are credited.

\section{References}

[1] J.S. Schwinger, On gauge invariance and vacuum polarization, Phys. Rev. 82 (1951) 664 [INSPIRE].

[2] M. Bleicher et al., Relativistic hadron hadron collisions in the ultrarelativistic quantum molecular dynamics model, J. Phys. G 25 (1999) 1859 [hep-ph/9909407] [InSPIRE].

[3] T. Hatsuda and T. Kunihiro, QCD phenomenology based on a chiral effective Lagrangian, Phys. Rept. 247 (1994) 221 [hep-ph/9401310] [INSPIRE].

[4] L. Álvarez-Gaumé and E. Witten, Gravitational anomalies, Nucl. Phys. B 234 (1984) 269 [INSPIRE].

[5] D.E. Kharzeev, L.D. McLerran and H.J. Warringa, The effects of topological charge change in heavy ion collisions: 'Event by event P and CP-violation', Nucl. Phys. A 803 (2008) 227 [arXiv: 0711.0950] [INSPIRE].

[6] K. Fukushima, D.E. Kharzeev and H.J. Warringa, The chiral magnetic effect, Phys. Rev. D 78 (2008) 074033 [arXiv: 0808.3382] [inSPIRE].

[7] D.E. Kharzeev and H.J. Warringa, Chiral magnetic conductivity, Phys. Rev. D 80 (2009) 034028 [arXiv:0907.5007] [INSPIRE]. 
[8] K. Fukushima, D.E. Kharzeev and H.J. Warringa, Electric-current susceptibility and the chiral magnetic effect, Nucl. Phys. A 836 (2010) 311 [arXiv:0912.2961] [INSPIRE].

[9] G. Basar, G.V. Dunne and D.E. Kharzeev, Chiral magnetic spiral, Phys. Rev. Lett. 104 (2010) 232301 [arXiv:1003.3464] [INSPIRE].

[10] D.E. Kharzeev and D.T. Son, Testing the chiral magnetic and chiral vortical effects in heavy ion collisions, Phys. Rev. Lett. 106 (2011) 062301 [arXiv: 1010.0038] [INSPIRE].

[11] STAR collaboration, B.I. Abelev et al., Azimuthal charged-particle correlations and possible local strong parity violation, Phys. Rev. Lett. 103 (2009) 251601 [arXiv:0909.1739] [INSPIRE].

[12] STAR collaboration, B.I. Abelev et al., Observation of charge-dependent azimuthal correlations and possible local strong parity violation in heavy ion collisions, Phys. Rev. C 81 (2010) 054908 [arXiv:0909.1717] [INSPIRE].

[13] A. Vilenkin, Cancellation of equilibrium parity violating currents, Phys. Rev. D 22 (1980) 3067 [INSPIRE].

[14] A. Vilenkin, Quantum field theory at finite temperature in a rotating system, Phys. Rev. D 21 (1980) 2260 [INSPIRE].

[15] D.T. Son and P. Surowka, Hydrodynamics with triangle anomalies, Phys. Rev. Lett. 103 (2009) 191601 [arXiv:0906.5044] [InSPIRE].

[16] J. Erdmenger, M. Haack, M. Kaminski and A. Yarom, Fluid dynamics of R-charged black holes, JHEP 01 (2009) 055 [arXiv:0809.2488] [INSPIRE].

[17] N. Banerjee et al., Hydrodynamics from charged black branes, JHEP 01 (2011) 094 [arXiv: 0809.2596] [INSPIRE].

[18] D.T. Son and A.R. Zhitnitsky, Quantum anomalies in dense matter, Phys. Rev. D 70 (2004) 074018 [hep-ph/0405216] [INSPIRE].

[19] V.P. Nair, R. Ray and S. Roy, Fluids, anomalies and the chiral magnetic effect: a group-theoretic formulation, Phys. Rev. D 86 (2012) 025012 [arXiv:1112.4022] [INSPIRE].

[20] J.-Y. Chen, D.T. Son, M.A. Stephanov, H.-U. Yee and Y. Yin, Lorentz invariance in chiral kinetic theory, Phys. Rev. Lett. 113 (2014) 182302 [arXiv:1404.5963] [INSPIRE].

[21] J.-H. Gao, Z.-T. Liang, S. Pu, Q. Wang and X.-N. Wang, Chiral anomaly and local polarization effect from quantum kinetic approach, Phys. Rev. Lett. 109 (2012) 232301 [arXiv: 1203.0725] [INSPIRE].

[22] G.E. Volovik, The Universe in a helium droplet, Int. Ser. Monogr. Phys. 117 (2006) 1.

[23] M. Neupane et al., Observation of a three-dimensional topological Dirac semimetal phase in high-mobility $\mathrm{Cd}_{3} \mathrm{As}_{2}$, Nature Commun. 5 (2014) 3786 [arXiv:1309.7892].

[24] Z.K. Liu et al., Discovery of a three-dimensional topological Dirac semimetal, $\mathrm{Na}_{3} \mathrm{Bi}$, Science 343 (2014) 864 [arXiv: 1310.0391].

[25] A.M. Turner and A. Vishwanath, Beyond band insulators: topology of semi-metals and interacting phases, arXiv:1301.0330 [INSPIRE].

[26] K. Iida and G. Baym, Superfluid phases of quark matter. 3. Supercurrents and vortices, Phys. Rev. D 66 (2002) 014015 [hep-ph/0204124] [INSPIRE]. 
[27] J.M. Leinaas and J. Myrheim, Intermediate statistics for vortices in superfluid films, Phys. Rev. B 37 (1988) 9286 [INSPIRE].

[28] M.M. Salomaa and G.E. Volovik, Quantized vortices in superfluid He-3, Rev. Mod. Phys. 59 (1987) 533 [Erratum ibid. 60 (1988) 573] [InSPIRE].

[29] V.P. Kirilin, A.V. Sadofyev and V.I. Zakharov, Chiral vortical effect in superfluid, Phys. Rev. D 86 (2012) 025021 [arXiv: 1203.6312] [InSPIRE].

[30] V.P. Kirilin, Z.V. Khaidukov and A.V. Sadofyev, Chiral vortical effect in Fermi liquid, Phys. Lett. B 717 (2012) 447 [arXiv:1203.6612] [INSPIRE].

[31] C. Xiong, M.R.R. Good, Y. Guo, X. Liu and K. Huang, Relativistic superfluidity and vorticity from the nonlinear Klein-Gordon equation, Phys. Rev. D 90 (2014) 125019 [arXiv: 1408.0779] [INSPIRE].

[32] T. Kalaydzhyan, Temperature dependence of the chiral vortical effects, Phys. Rev. D 89 (2014) 105012 [arXiv:1403.1256] [INSPIRE].

[33] P.V. Buividovich, Axial magnetic effect and chiral vortical effect with free lattice chiral fermions, arXiv: 1309.4966 [INSPIRE].

[34] D.-F. Hou, H. Liu and H.-c. Ren, A possible higher order correction to the vortical conductivity in a gauge field plasma, Phys. Rev. D 86 (2012) 121703 [arXiv:1210.0969] [INSPIRE].

[35] S. Golkar and D.T. Son, Non-renormalization of the chiral vortical effect coefficient, arXiv:1207.5806 [INSPIRE].

[36] K. Landsteiner, E. Megias, L. Melgar and F. Pena-Benitez, Holographic gravitational anomaly and chiral vortical effect, JHEP 09 (2011) 121 [arXiv:1107.0368] [INSPIRE].

[37] K. Landsteiner, E. Megias and F. Pena-Benitez, Frequency dependence of the chiral vortical effect, Phys. Rev. D 90 (2014) 065026 [arXiv: 1312.1204] [INSPIRE].

[38] R. Loganayagam and P. Surowka, Anomaly/transport in an ideal Weyl gas, JHEP 04 (2012) 097 [arXiv: 1201.2812] [INSPIRE].

[39] K. Jensen, P. Kovtun and A. Ritz, Chiral conductivities and effective field theory, JHEP 10 (2013) 186 [arXiv: 1307.3234] [INSPIRE].

[40] R. Baier, P. Romatschke, D.T. Son, A.O. Starinets and M.A. Stephanov, Relativistic viscous hydrodynamics, conformal invariance and holography, JHEP 04 (2008) 100 [arXiv: 0712.2451] [INSPIRE].

[41] G.D. Moore and K.A. Sohrabi, Kubo formulae for second-order hydrodynamic coefficients, Phys. Rev. Lett. 106 (2011) 122302 [arXiv: 1007.5333] [INSPIRE].

[42] P. Arnold, D. Vaman, C. Wu and W. Xiao, Second order hydrodynamic coefficients from 3-point stress tensor correlators via AdS/CFT, JHEP 10 (2011) 033 [arXiv:1105.4645] [INSPIRE].

[43] G.D. Moore and K.A. Sohrabi, Thermodynamical second-order hydrodynamic coefficients, JHEP 11 (2012) 148 [arXiv:1210.3340] [INSPIRE].

[44] N. Banerjee et al., Constraints on fluid dynamics from equilibrium partition functions, JHEP 09 (2012) 046 [arXiv:1203.3544] [INSPIRE].

[45] J. Bhattacharya, S. Bhattacharyya and M. Rangamani, Non-dissipative hydrodynamics: effective actions versus entropy current, JHEP 02 (2013) 153 [arXiv:1211.1020] [INSPIRE]. 
[46] K. Jensen et al., Towards hydrodynamics without an entropy current, Phys. Rev. Lett. 109 (2012) 101601 [arXiv:1203.3556] [INSPIRE].

[47] B.S. Dewitt, The global approach to quantum field theory, Oxford University Press, Oxford U.K. (2003).

[48] S.A. Fulling, Aspects of quantum field theory in curved space-time, London Mathematical Society student texts volume 17, Cambridge University Press, Cambridge U.K. (1989).

[49] C. Itzykson and J.B. Zuber, Quantum field theory, Mcgraw-Hill, New York U.S.A. (1980).

[50] B.Z. Iliev, Lagrangian quantum field theory in momentum picture. II. Free spinor fields, hep-th/0405008 [INSPIRE].

[51] T. Kugo, Eichtheorie, Springer, Berlin Germany (1997).

[52] A. Adams, P.M. Chesler and H. Liu, Holographic turbulence, Phys. Rev. Lett. 112 (2014) 151602 [arXiv:1307.7267] [INSPIRE]. 